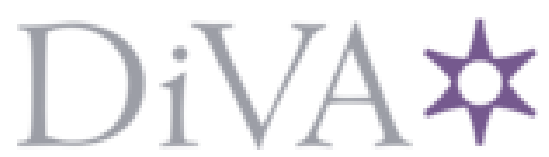

http://www.diva-portal.org

This is the published version of a paper published in Journal of Applied Physics.

Citation for the original published paper (version of record):

Davidenko, N., Davidenko, I., Savchenko, I., Popenaka, A., Bååth, L. (2008)

Electro-optical effect in films of azobenzene polycomplexes with cobalt.

Journal of Applied Physics, 103(9): Article number 094323

http://dx.doi.org/10.1063/1.2913315

Access to the published version may require subscription.

N.B. When citing this work, cite the original published paper.

Permanent link to this version:

http://urn.kb.se/resolve?urn=urn:nbn:se:hh:diva- 1884 


\title{
Electro-optical effect in films of azobenzene polycomplexes with cobalt
}

\author{
N. A. Davidenko, ${ }^{1, a)}$ I. I. Davidenko, ${ }^{1}$ I. A. Savchenko, ${ }^{1}$ A. N. Popenaka, ${ }^{1}$ and \\ L. B. Baath ${ }^{2}$ \\ ${ }^{1}$ Kiev Taras Shevchenko National University, Volodymyrska Str., 60, 01033, Kiev-33, Ukraine \\ ${ }^{2}$ Halmstad University, SE-31018, Halmstad, Sweden
}

(Received 26 October 2007; accepted 28 February 2008; published online 14 May 2008)

Polymeric films containing azobenzene fragments with donor and acceptor groups were produced as well as films of polycomplexes of these fragments with cobalt. Absorption spectra of these films before and during application of an external electric field were investigated. Electro-optical properties of the films are conditioned by spatial reorientation of dipole moments of azobenzene groups induced by polarized light in an external electric field. Increase in dipole moments of azobenzene groups reduces the influence of cobalt ions on the electro-optical properties of the polycomplex films. In the proposed phenomenological model, the electro-optical effect is explained by effect of induced forces upon azobenzene isomers and metallic ions. (C) 2008 American Institute of Physics. [DOI: 10.1063/1.2913315]

\section{INTRODUCTION}

Films of polymeric compositions (PCs) containing azobenzene dyes $^{1-3}$ or azobenzene lateral groups ${ }^{4-6}$ are of interest for their application as optically active media, in particular, as polarization sensitive media for optical holographic recording. Induced polarization appears in the PC films under the influence of linearly polarized light which is absorbed by the azobenzene groups and causes changes of isomeric structures. The photoinduced polarization can be conserved at room temperature during quite a long time. Changes of the polarization are possible under the influence of external thermal or mechanical treatments, illumination with light and in external electric or/and magnetic fields. Rotation of the dipole moments happens in an external electric field. PC can be prepared either on the base of metal containing monomers ${ }^{7}$ or with additions of magnetic nanoparticles $^{8}$ to intensify the magnetic field influence. Since the mechanism of external electric and magnetic influences is determined by the induced forces orienting the dipole moments of the azobenzene compounds, one can suppose that the electro-optical effects could be revealed in PC with azobenzene groups and metallic ions chemically connected to the polymer. However, the electro-optical effect in such PC depending on presence of donor or acceptor groups in azobenzene structure has not been investigated so far. These groups can increase or decrease dipole moment of the azobenzene isomers. This fact is important for proper choice of compounds designed for application in optoelectronic devices. Thus, investigations of electro-optical properties of PC films based on azobenzene with donor and acceptor groups depending on presence of metallic ions in their structure as well as development of an adequate physical model of the electro-optical effect is the aim of the present work.

\footnotetext{
a)Electronic mail: daviden@ukrpack.net.
}

\section{INVESTIGATED OBJECTS AND EXPERIMENTAL TECHNIQUE}

The following structural analogs were synthesized for the investigations: 4-methacryloyloxy-2- $(N, N$ diethylamino)-(4' - carboxy-3' -oxy)azobenzene (A1) containing donor group in the azobenzene fragment; the polycomplex (A1-Co); 4-methacryloyloxy-(4' -carboxy-3' oxy)azobenzene (A2); the polycomplex (A2-Co); 4-methacryloyloxy-2-nitro-(4' -carboxy-3' -oxy)azobenzene (A3) containing acceptor nitrogroup in the azobenzene fragment; the polycomplex (A3-Co):

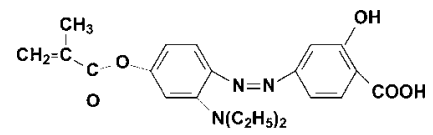

AI
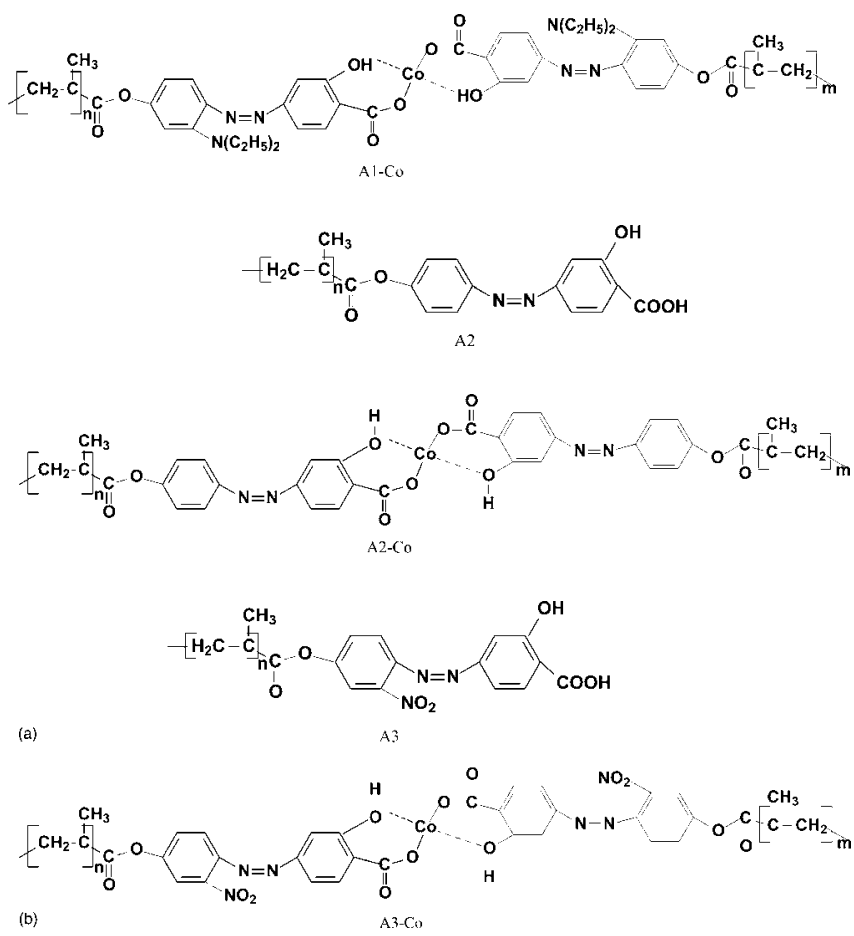


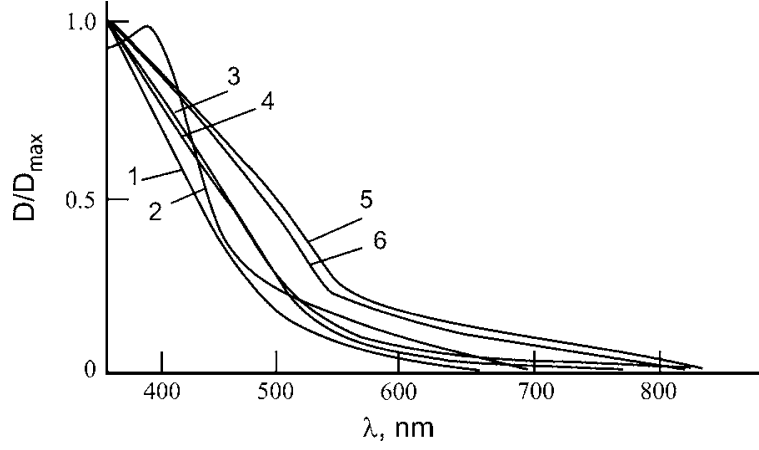

FIG. 1. The spectra of the optical density of films A1 (1), A1-Co (2), A2 (3), A2-Co (4), A3 (5), and A3-Co (6).

The samples for investigations were prepared as structures with a free surface: a glass substrate-a conducting layer $\mathrm{SnO}_{2}: \mathrm{In}_{2} \mathrm{O}_{3}-$ a PC film (1-2 $\left.\mu \mathrm{m}\right)$. Spectra of optical density $(D)$ of the PC films over the range of light wavelength $\lambda=350-900 \mathrm{~nm}$ were measured in these samples as well as the value $\delta I_{E}=\left(I_{E}-I_{0}\right) / I_{0}$, where $I_{0}$ and $I_{E}$ are, respectively, the intensities of monochromatic light passed through the sample before and after external electric field is applied. Unpolarized as well as polarized light was used for illumination of the samples. In the second case, a sample was placed between two polarizers. The electric field $E=1$ $\times 10^{8} \mathrm{~V} / \mathrm{m}$ in the PC film was produced by a crown discharge in a special device. The dependencies of $\delta I_{E}$ on time $t$ of the sample illumination, on time after this illumination, on light wavelength $\lambda$ corresponding to long wave edge of the PC films absorption, on angle $\theta$ between axes of the polarizer and the analyzer were measured. All experiments were carried out at the temperature $T=293 \mathrm{~K}$ when depolarizing influence of $\mathrm{SnO}_{2}: \mathrm{In}_{2} \mathrm{O}_{3}$ layer is negligible.

\section{RESULTS}

The absorption spectra of the investigated PC films are shown in Fig. 1. Over the visible range, the absorption is conditioned by photoexcitation of azobenzene groups, and it weakly depends on presence of metallic ions in PC. The bathochromic shift in the series (A1,A1-Co) $\rightarrow(\mathrm{A} 2, \mathrm{~A} 2-\mathrm{Co}) \rightarrow(\mathrm{A} 3, \mathrm{~A} 3-\mathrm{Co})$ is caused by increase of chromophore dipole moment when acceptor nitrogroup is present instead of donor group as well as by lowering the energy of excited states of organic dyes. ${ }^{10,11}$

The intensity of unpolarized light passed through the sample with investigated PC film decreases (Fig. 2) after external electric field is applied, it returns to the initial state

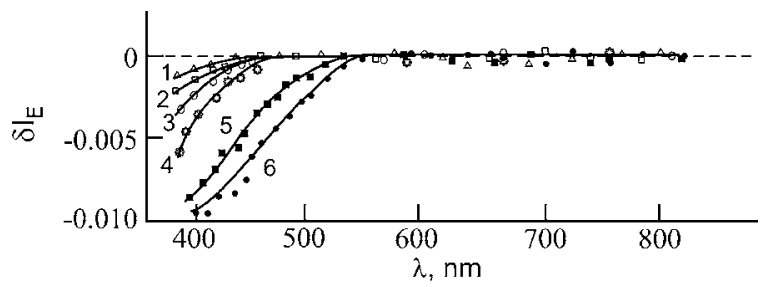

FIG. 2. The dependencies of $\delta I_{E}$ on $\lambda$ measured under the influence of unpolarized light in the virgin samples with films A1 (1), A1-Co (2), A2 (3), A2-Co (4), A3 (5), and A3-Co (6).

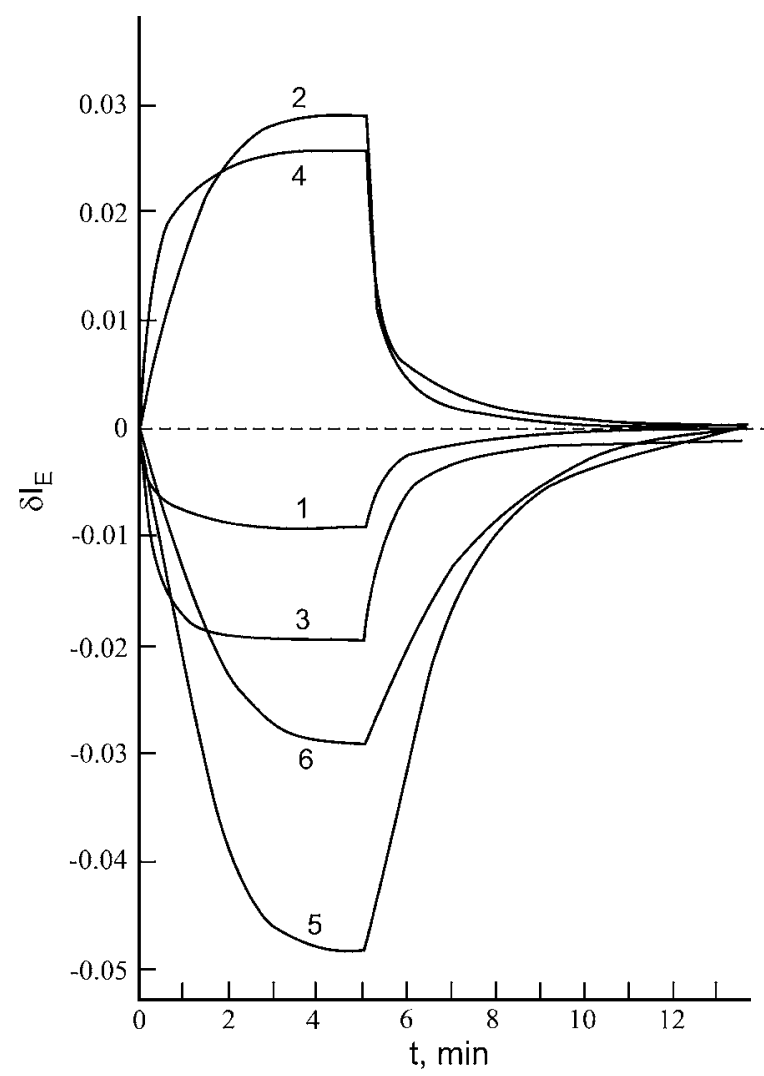

FIG. 3. The dependencies of $\delta I_{E}$ on time $t$ after application of the external electric field and its switching off after 5 min measured for $\theta=\pi / 2$ and $\lambda$ $=544 \mathrm{~nm}$ in the samples with films A1 (1), A1-Co (2), A2 (3), A2-Co (4), A3 (5), and A3-Co (6). The samples were preliminarily illuminated with linearly polarized light $(\lambda<500 \mathrm{~nm})$ for $60 \mathrm{~min}$.

when the field is switched off. It is well known that at the room temperature in PC similar to investigated in the present paper the trans-state of azobenzene groups is predominant before illumination with linearly polarized light. Under an influence of linearly polarized light in these PC induced polarization appears caused by the changes of the concentrations of the trans- and cis-isomers of the azobenzene groups. Orientations of the electric dipole moments of trans- and cis-isomers of the azobenzene groups do not coincide. These orientations also do not coincide with the corresponding dipole moments of the optical transitions. Therefore, increase of unpolarized light absorption by the trans-isomers of the azobenzene groups in the PC when an external electric field is applied (Fig. 2) is caused by a rotation of the electric dipole moments of the azobenzene groups according to the field direction and by a respective rotation of the dipole moments of the optical transitions of these azobenzene groups. Since the isomers of the azobenzene groups are mixed in the polymeric binder and are chemically connected to the main polymeric chain (in our case), slowness of the azobenzene groups rotation after an external electric field application and switching off is quite natural. This fact is demonstrated by Fig. 3 where the experimental dependencies of the light intensity passed through PC under an influence of an external electric field and after its ceasing are present. The kinetics of the light intensity changes from $I_{0}$ to $I_{E}$ and after the field is switched off (Fig. 3) can be described by following simplified expressions: 


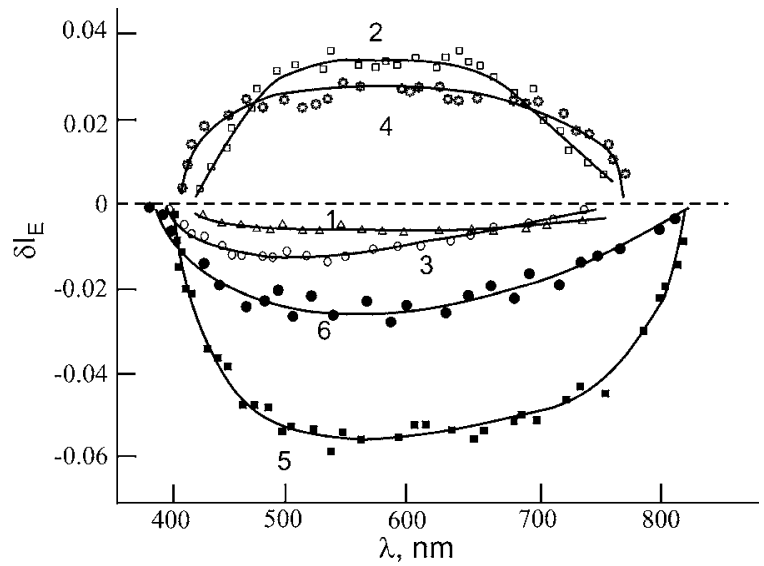

FIG. 4. The dependencies of $\delta I_{E}$ on $\lambda$ measured for $\theta=\pi / 2$ in the samples with A1 (1), A1-Co (2), A2 (3), A2-Co (4), A3 (5), and A3-Co (6) after their previous illumination with linearly polarized light $(\lambda<500 \mathrm{~nm})$ for $60 \mathrm{~min}$

$$
\begin{aligned}
& I(t)=I_{0}+\left(I_{E}-I_{0}\right)\left[1-\exp \left(-t / \tau_{E}\right)\right], \\
& \left.I(t)=I_{0}+\left(I_{E}-I_{0}\right) \exp \left[-t / \tau_{E}\right)\right],
\end{aligned}
$$

where the time constant $\tau_{E}$ is $60 \pm 10 \mathrm{~s}$ for $\mathrm{A} 2$ and $\mathrm{A} 2-\mathrm{Co}$, $130 \pm 10 \mathrm{~s}$ for $\mathrm{A} 1$ and $\mathrm{A} 1-\mathrm{Co}$, and $150 \pm 10 \mathrm{~s}$ for $\mathrm{A} 3$ and A3-Co. Our experiments do not allow to determine the dependency of $\tau_{E}$ on $E$ because the field in the PC with the free surface was formed by the corona charging device with the stable field strength. The devices of such type create in the PC the field $E$ of maximal value. Investigations of the dependency $\tau_{E}(E)$ should be done in the PC samples of the sandwich structure with metallic contacts. This is a subject of our following investigations. Through the investigated PC series growth of $\tau_{E}$ at $E=10^{8} \mathrm{~V} / \mathrm{m}$ was observed. It can be explained by steric factors affecting on rotation of the isomers of the azobenzene fragments with donor and acceptor groups. The electric field influence is more essential for A3, A3-Co as compared to A1, A1-Co and A2, A2-Co. It decreases under $\lambda$ growth and it is not observed for $\lambda$ $>560 \mathrm{~nm}$.

The field influence on the light transmission through the investigated samples is more significant for samples that were preliminarily illuminated with polarized light with wavelength $\lambda<550 \mathrm{~nm}$ from the absorption range of the azobenzene groups. Illumination of the PC with unpolarized light from the absorption range of the trans-isomers of the azobenzene groups results in a transformation of these transstates into the cis-states. This is the reason of appearance of the photoinduced optical anisotropy in the PC. However, the dipole moments of the optical transitions for the trans- and cis-states of the azobenzene groups do not coincide. This fact causes a difference between effects of the influence of an external electric field on transmission of polarized and unpolarized light through the investigated PC. The most intensive changes of $I_{0}$ in applied electric field happen after illumination of the samples with polarized light for $t>30 \mathrm{~min}$ for analyzer angle $\theta=\pi / 2 .^{12}$ The experimental spectral dependencies of $\delta I_{E}$ after quite a long illumination of the samples with polarized light with $\lambda<550 \mathrm{~nm}$ are shown in Fig. 4 $(\theta=\pi / 2)$. Comparing dependencies shown in Figs. 1, 2, and
4, one can conclude that after long illumination of the samples with linearly polarized light, the field influence on the light transmission gains strength within the long wave range of PC absorption spectra. The time constants of the light intensity changes after applying field and ceasing it determined from time dependencies of $\delta I_{E}$ (Fig. 3) are close to $\tau_{E}$ in the expressions (1) and (2) for the case of unpolarized light.

\section{DISCUSSION}

In the investigated PC samples, the photoinduced optical anisotropy arises as a result of illumination with a linearly polarized light. trans-cis isomerization of the azobenzene groups happens under such light influence. An external electric field has orienting effect on the photoinduced dipole moments of these groups. This effect reveals itself in appearance of electro-optical effect over the range of light wavelength corresponding to the long wave edge of PC absorption spectra. The influence of electric field on the PC films absorption even without preliminary illumination with linearly polarized light (Fig. 2) is also connected with rotation of the dipole moments of the azobenzene groups.

In films A1, A2, and A3-Co, an external electric field provokes alignment of the photoinduced dipoles along the force lines of the field. As a result, interaction between polarized light and these dipoles is weaker and $\delta I_{E}<0$ for $\theta$ $=\pi / 2$. In films A1-Co and A2-Co in the external electric field, polarized light undergoes more intensive dispersion and depolarization resulting in positive $\delta I_{E}$. This peculiarity is probably caused by more effective interaction between the $\mathrm{Co}^{2+}$ ions and the electric field as compared to dipoles interaction with the field. These ions are bonded to the azobenzene groups indirectly, and the direction of the bond and the photoinduced dipole moment do not coincide. Hence, change of orientation of the photoinduced dipoles bonded to the metallic ions in the samples with A1-Co, A2-Co, and A3-Co in the external electric field could be opposite to the change in the samples with A1, A2, and A3. The influence of the metallic ions in the samples with A3-Co is less than in A1-Co and A2-Co due to increased dipole moment of the azobenzene group, rotation of this dipole moment in the external electric field dominates.

\section{MODEL}

To explain the described experimental results, a phenomenological model based on analysis of equilibrium configuration of the azobenzene group isomer and connected ion $\mathrm{Co}^{2+}$ in the external electric field (Fig. 5) was developed. In Fig. 5(d) is the dipole moment of the azobenzene group isomer $r_{1}$. It consists of two similar benzene rings which are symmetrical, respectively, to the center of $\mathrm{N}-\mathrm{N}$ bond in trans-state and symmetrical, respectively, to the plane transient perpendicularly through the center of $\mathrm{N}-\mathrm{N}$ bond in cisstate. Therefore, the dipole moments of two benzene parts get balanced in the first case and are summed up in the second case. In the second case, the total dipole moment $\mathbf{d}$ is situated in the symmetry plane perpendicularly to the molecular chain $r_{1}$. The radius vectors $\mathbf{r}_{2}, \mathbf{r}_{3}$, and $\mathbf{r}_{4}$ describe 


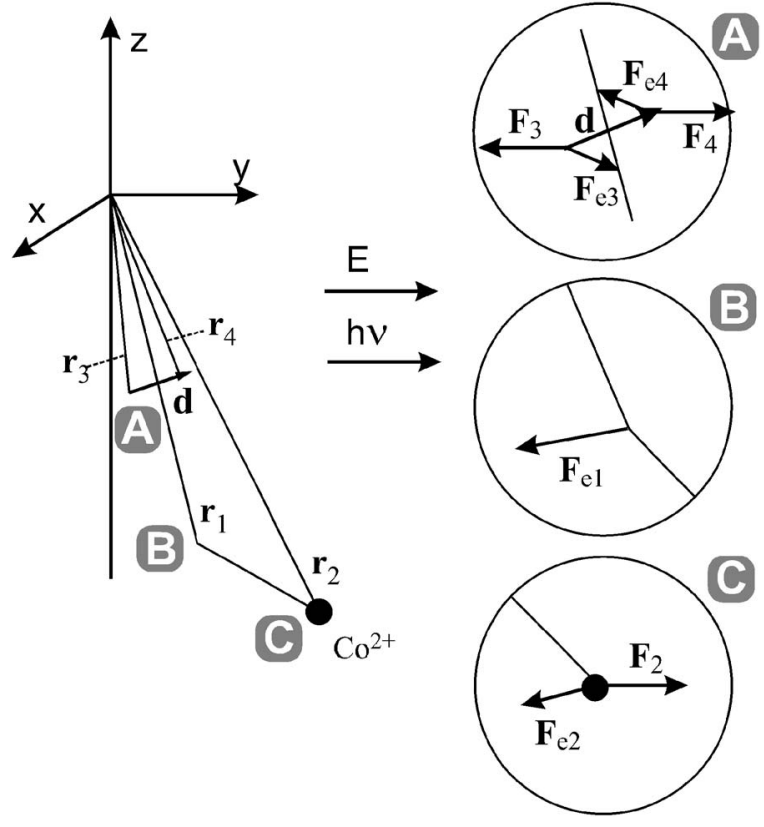

FIG. 5. The model of the influence of external electric field on the dipole moment of the azobenzene group and bonded to it $\mathrm{Co}^{2+}$ ion for compounds A1-Co and A3-Co.

positions of $\mathrm{Co}^{2+}$ ion and the dipole moment of the azobenzene group isomer, respectively. The electric field $\mathbf{E}$ effects on the $\mathrm{Co}^{2+}$ ion through the force $\mathbf{F}_{2}$ and on the dipole moment $\mathbf{d}$ through the forces pair $\mathbf{F}_{3}$ and $\mathbf{F}_{4}$. The restoring forces $\mathbf{F}_{e 1}$ (on the molecular chain of the azobenzene group isomer), $\mathbf{F}_{e 2}\left(\right.$ on $\mathrm{Co}^{2+}$ ) and forces pair $\mathbf{F}_{e 3}$ and $\mathbf{F}_{e 4}$ (on the dipole moment of the azobenzene group isomer) appear in the considered system as well. For the small declinations from the equilibrium state, these forces can be considered as being proportional to shift of the corresponding radius vector from the initial state $\mathbf{r}_{i 0}$ to the state $\mathbf{r}_{i}: \mathbf{F}_{e i}=k_{i}\left(\mathbf{r}_{i 0}-\mathbf{r}_{i}\right)$, where $k_{i}$ characterizes the quasielastic forces appearing in the molecule due to shift of its nuclei from their equilibrium states.

The equilibrium state of the azobenzene group and connected $\mathrm{Co}^{2+}$ ion is described by the following set of vector equations:

$$
\begin{aligned}
& \mathbf{F}_{e 1}+\mathbf{F}_{e 2}+\mathbf{F}_{e 3}+\mathbf{F}_{e 4}+\mathbf{F}_{2}+\mathbf{T}=0, \\
& \mathbf{M}_{2}+\mathbf{M}_{3}+\mathbf{M}_{4}+\mathbf{M}_{e 1}+\mathbf{M}_{e 2}+\mathbf{M}_{e 3}+\mathbf{M}_{e 4}=0,
\end{aligned}
$$

where $\mathbf{M}_{\mathbf{i}}=\left[\mathbf{r}_{\mathbf{i}} \times \mathbf{F}_{\mathbf{i}}\right], \mathbf{M}_{e i}=\left[\mathbf{r}_{i} \times \mathbf{F}_{e i}\right], \mathbf{T}$ is the external force appearing in the point $(0,0,0)$ of fixation of the system.

In chosen geometric configuration (Fig. 5), experimentally registered changes of depolarizing properties of the investigated films should be attributed to appearance of the component $d_{x}$ of the dipole moment of the azobenzene group isomer. The initial state with the dipole moment in the plane $Y 0 Z$ is interesting for numerical simulation. For example, such state can be reached by previous illumination with linearly polarized light with the wave vector $\mathbf{k} \| 0 Y$ and polarization vector $\mathbf{E} \| 0 Z$. In this initial state, influence of external electric field on $\mathrm{A} 1-\mathrm{A} 3$ is absent because in these compounds the dipole moments of the azobenzene groups isomer are already oriented along the field direction as a result of illumination with linearly polarized light. On the contrary, in

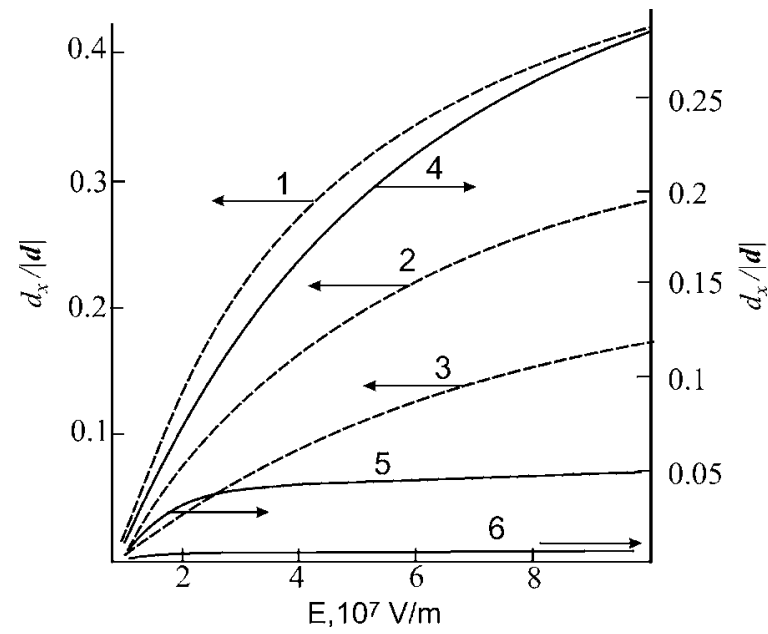

FIG. 6. The calculated dependencies of the $X$ component of the dipole moment of the azobenzene group on $E$ for $|\mathbf{d}|=0.1 d_{0}$ and $\eta=0.5$ (1), 1 (2), $2(3) ; \eta=1$ and $|\mathbf{d}|=0.1 d_{0}(4), d_{0}(5), 10 d_{0}(6)$. The value of $1 \mathrm{D}$ was used as the parameter $d_{0}$.

the compounds A1-Co-A3-Co, the field involves rotation of the azobenzene group and connected ion accompanying by inclination of the dipole moment from $Y 0 Z$ plane due to absence of collinearity between the molecular chain and direction of connection of $\mathrm{Co}^{2+}$ to it.

The set of Eq. (3) was solved numerically, the dependencies of $X$ component of the normalized dipole moment $d_{x} /|\mathbf{d}|$ on the strength of external electric field $E$ are shown in Fig. 6 . The curves 1,2, and 3 were calculated for different values of ratio between coefficients of quasielastic forces $\eta=k_{2} / k_{1}$ $\left(k_{3}=k_{4}=0.01 k_{1}\right)$, the curves 4,5 , and 6 correspond to different values of the dipole moment $|\mathbf{d}|$. By analyzing these results, one can conclude that the inclination of the dipole moment from the $Y O Z$ plane and hence, experimentally registered changes of passing light depolarization decrease under increase of both, the value of dipole moment and $\eta$. Growth of $\eta$ corresponds to increase of quasielastic force which hinders rotation of the dipole moment and its inclination from the plane $Y 0 Z$. The influence of interaction of $\mathrm{Co}^{2+}$ ion with electric field is less in the compounds with increased dipole moment. We supposed that this interaction is responsible for the inclination of the molecular chains from the $Y 0 Z$ plane. Therefore, growth of the dipole moments involves decrease of light depolarization.

The results of calculation within the scope of proposed phenomenological model confirm the following suppositions about the reasons of difference between the experimentally observed depolarization effects in the investigated polymeric compositions:

- In A1, external electric field contributes to alignment of the dipole moments of the azobenzene groups formed after preliminary illumination with linearly polarized light in the plane $Y 0 Z$; as a result, depolarization of passing light decreases.

- In A1-Co with small dipole moment of the azobenzene group, isomer interaction of $\mathrm{Co}^{2+}$ ion with electric field dominates; as a result, inclination of the molecular chain of the azobenzene group from the $Y 0 Z$ plane 
happens due to absence of collinearity between bones; depolarization of passing light is observed.

- A3 possesses increased dipole moment of the azobenzene group as compared to A1; thus, external electric field has more essential aligning influence in this case, more sufficient decrease of light depolarization occurs in external electric field.

- In A3-Co, presence of $\mathrm{Co}^{2+}$ ions provokes inclination of the molecular chain from the $Y 0 Z$ plane in the field and respective depolarization but increased dipole moment of the azobenzene group provides its orientation along the field direction; as a result, the influence of $\mathrm{Co}^{2+}$ ion becomes not essential.

If, for considered geometric configuration (Fig. 5), the medium was illuminated with linearly polarized light with $\mathbf{k} \| 0 Z$ during quite long time, then the dipole moments of the azobenzene groups are mostly oriented in the plane $X 0 Y$ because, in this case, the probability of their interaction with light is minimal. Monte-Carlo modeling allows to calculate the values $\left\langle d_{x} /|\mathbf{d}|\right\rangle$ for the compounds A1, A1-Co, and A2-Co under supposition of normal distribution of inclination of the dipole moments from the plane. For equal conditions and parameters of calculation, these values have different signs for A1, A2-Co, and A1-Co. This fact testifies as well the adequacy of above made suppositions about the physical mechanisms of the observed effects.

\section{CONCLUSION}

From the present results, one can conclude that the films of the azobenzene polycomplexes with metallic ions can be considered as attractive for application in information media sensitive for external electromagnetic influences. Media for electro-optical and magneto-optical light modulators ${ }^{13}$ as well as for polarization holography ${ }^{14,15}$ can exemplify such media. In these media, the effect of a constant external elec- tric field on a light transmission was attributed to displacement of the azobenzene groups and metallic ions from their equilibrium state under the field influence. Presence of the nitrogroup involves increase in the dipole moment of the azobenzene group, strengthens this effect and diminishes the influence of metallic ions on the rotation of the dipole moments of the azobenzene group. The results of the present investigation seem to be important for development of information media.

${ }^{1}$ S. K. Yesodha, C. K. S. Pillai, and N. Tsutsumi, Prog. Polym. Sci. 29, 45 (2004).

${ }^{2}$ J. C. Ribierre, G. Cheval, F. Huber, L. Mager, A. Fort, R. Muller, S. Mery, and J. F. Nicoud, J. Appl. Phys. 91, 1710 (2002).

${ }^{3}$ K. Janus, K. Matczyszyn, J. Sworakowski, J. F. Biernat, and Z. Galewski, Mol. Cryst. Liq. Cryst. Sci. Technol., Sect. A 361, 143 (2001).

${ }^{4}$ O. Yaroshchuk, A. Tereshchenko, Yu. Zakrevskyy, and I. Shanski, Mol. Cryst. Liq. Cryst. Sci. Technol., Sect. A 361, 187 (2001).

${ }^{5}$ L. Andruzzi, A. Altomare, F. Ciardelli, R. Solaro, S. Hvilsted, and P. S. Ramanujam, Macromolecules 32, 448 (1999).

${ }^{6}$ X. Zhou, H. Ren, C. Chen, and M. Wang, J. Appl. Polym. Sci. 73, 1913 (1999).

${ }^{7}$ G. K. Lebedeva, V. N. Ivanova, S. A. Ivanov, V. A. Lukoshkin, N. N. Smirnov, I. M. Sokolova, L. V. Khokhlova, and V. V. Kudryavtsev, Phys. Solid State 46, 569 (2004)

${ }^{8}$ A. A. Borsch, M. S. Brodyn, V. I. Volkov, V. R. Lyakhovetskii, and A. S. Kutsenko, Quantum Electron. 33, 441 (2003).

${ }^{9}$ N. Davidenko, V. Pavlov, N. Chuprina, I. Davidenko, and L. Baath, J. Appl. Phys. 100, 023111 (2006).

${ }^{10}$ Y. Wu, A. Natansohn, and P. Rochon, Macromolecules 34, 7822 (2001).

${ }^{11}$ N. A. Davidenko, A. A. Ishchenko, and N. G. Kuvshinsky, Photonics of Molecular Semiconductor Composites Based on Organic Dyes (Naukova Dumka, Kiev, 2005).

${ }^{12}$ I. I. Davidenko, N. A. Davidenko, I. A. Savchenko, and V. G. Syromyatnikov, J. Appl. Spectrosc. 72, 541 (2005).

${ }^{13}$ I. I. Davidenko, N. A. Davidenko, I. A. Savchenko, and V. G. Syromyatnikov, Ukrainian Patent No. 77498 (October 2006).

${ }^{14} \mathrm{H}$. Ono and N. Kawatsuki, Advances in Technology of Materials and Materials Processing 7, 9 (2005).

${ }^{15}$ N. A. Davidenko, I. I. Davidenko, A. N. Popenaka, I. A. Savchenko, and A. N. Shumelyuk, J. Appl. Spectrosc. 74, 830 (2007). 
Journal of Applied Physics is copyrighted by the American Institute of Physics (AIP). Redistribution of journal material is subject to the AIP online journal license and/or AIP copyright. For more information, see http://ojps.aip.org/japo/japcr/jsp 